\title{
INFARTO AGUDO DE MIOCARDIO CON ELEVACIÓN DEL SEGMENTO ST: PREVALENCIA DE ARTERIA SEGÚN EL SEXO
}

\author{
ST-SEGMENT ELEVATION MYOCARDIAL INFARCTION: PREVALENCE OF \\ ARTERY ACCORDING TO GENDER
}

\author{
ALFREDO ASTESIANO ${ }^{1}$, NICOLÁS F. RENNA², MARÍA J. TORRES ${ }^{3}$, RODRIGO GARCÍA4 ${ }^{4}$, MARIANO GRUCCI ${ }^{5}$, ALBERTO G. VILLASÍS $^{5}$, \\ MARCIA ZULOAGA PUGLISI ${ }^{5}$, ROMANO M. TRIONFI ${ }^{6}$
}

\section{RESUMEN}

Introducción. A pesar de los grandes avances en el conocimiento de la fisiopatología del infarto agudo de miocardio con elevación del segmento ST (IAMCEST), aún se desconocen variables que influyen en el desenlace de esta patología y en particular la relación entre la anatomía coronaria afectada y el sexo. En Argentina existe información limitada sobre este aspecto.

Objetivo. Analizar las características clínicas de pacientes con IAMCEST en un centro privado de la provincia de Mendoza, con la intención de evaluar la arteria coronaria afectada según el sexo.

Métodos. Estudio observacional y retrospectivo, de pacientes que ingresaron a nuestro centro con diagnóstico de IAMCEST, en los que se les practicó cinecoronariografía de urgencia. Se analizaron variables clínicas como sexo, edad, factores de riesgo cardiovascular, arteria culpable, tipo de tratamiento y stent colocado. Resultados. Se incluyeron 153 pacientes. La edad media fue 70 años $( \pm 13)$ en mujeres y $61( \pm 12)$ en hombres. El factor de riesgo cardiovascular más prevalente en ambos grupos fue la hipertensión arterial, siendo $79,5 \%$ en mujeres y $61,5 \%$ en hombres ( $p=0,04)$; seguido por tabaquismo ( $35,9 \%$ mujeres y $53 \%$ hombres), diabetes mellitus $(35,9 \%$ mujeres y $21,4 \%$ hombres; $p=0,07)$ y obesidad $(28,2 \%$ mu jeres y $29,1 \%$ hombres; $p=0,91)$. No hubo diferencias de la arteria afectada según sexo $(p=0,38)$. La arteria culpable más prevalente fue la descendente anterior (DA) $39,5 \%$ en mujeres y $37,9 \%$ en hombres). Resultados similares se observaron con la arteria coronaria derecha, con una prevalencia ligeramente menor que la DA (34,2\% mujeres y $35,3 \%$ hombres); la arteria circunfleja fue el vaso culpable en $18,42 \%$ sexo femenino y $17,2 \%$ sexo masculino, El $2,6 \%$ de las mujeres tuvo compromiso de tronco de la arteria coronaria izquierda $(\mathrm{TCl})$, siendo mayor el porcentaje de hombres con compromiso de este vaso: $6 \%$. El puente venoso tuvo una baja prevalencia sin diferencias entre sexo (2,6\% mujeres y 3,4\% hombres).

Conclusión. Más del $75 \%$ de los infartos de miocardio con elevación del segmento ST fueron hombres, sin embargo las mujeres presentaron un perfil de riesgo cardiovascular diferente, siendo más prevalente la HTA y la edad avanzada. No hubo diferencias en la arteria coronaria afectada según el sexo, observando una tendencia no significativa en mayor enfermedad de TCl en el hombre.

Palabras clave: infarto de miocardio, sexo, vasos coronarios.

\section{ABSTRACT}

Introduction. Despite the great advances in the knowledge of the pathophysiology of acute myocardial infarction with ST-segment elevation (STEMI), there are still unknown variables that influence the outcome of this pathology and in particular the relationship between the affected coronary anatomy and gender. In Argentina there is limited information on this aspect.

Objective. To analyze the clinical characteristics of patients with STEMI in a private center in the province of Mendoza, with the intention of evaluating the affected coronary artery according to gender.

Methods. Observational and retrospective study of patients admitted to our center with a diagnosis of STEMI, in which they underwent emergency coronary angiography. We analyzed clinical variables such as gender, age, cardiovascular risk factors, culprit artery, type of treatment and stent placed.

Results. 153 patients were included. The mean age was 70 years $( \pm 13)$ in women and $61( \pm 12)$ in men. The most prevalent cardiovascular risk factor in both groups was hypertension, being $79.5 \%$ in women and $61.5 \%$ in men $(p=0.04)$; followed by smoking (35.9\% women and $53 \%$ men), diabetes mellitus ( $35.9 \%$ women and $21.4 \%$ men, $p=0.07)$ and obesity $(28.2 \%$ women and $29.1 \%$ men; $p=0.91)$. There were no differences in terms of the affected artery according to gender $(p=0.38)$. It was observed that the most prevalent culprit artery was the anterior descending artery (ADA), being $39.5 \%$ in women and $37.9 \%$ in men. Similar results were observed with the right coronary artery, with a slightly lower prevalence than the ADA (34.2\% women and $35.3 \%$ men); circumflex artery was the culprit vessel in $18.42 \%$ female sex and $17.2 \%$ male sex, $2.6 \%$ of women had a compromise of the left main coronary artery (LMCA), the percentage of men with compromise being higher: $6 \%$. Vein grafts had a low prevalence without gender differences (2.6\% women and $3.4 \%$ men).

Conclusion. More than $75 \%$ of myocardial infarctions with ST-segment elevation were men; however, women presented a cardiovascular risk profile different from men, with hypertension and older age being more prevalent. There were no differences in the coronary artery affected according to gender, observing a non-statistically significant trend in greater LMCA disease in men.

Keywords: myocardial infarction, sex, coronary vessels.

REVISTA CONAREC 2018;33(147):314-316 | DOI:10.32407/RCON/2018147/0314-0316

1. Jefe de Unidad Coronaria

2. Coordinador de Unidad Coronaria

3. Jefa de residentes de Cardiología

4. Ex Jefe de Residentes de Cardiología

5. Médico residente de Cardiología

6. Médico concurrente Cardiología. Hospital Español de Mendoza

$\triangle$ Correspondencia: Dra. Romano María Trionfi. Dirección: O’Higgins 1844. M5504PIR Godoy Cruz, Mendoza. Rep. Argentina. Tel: (261)-152180381. rtrionfi@hotmail.com.

Los autores declaran no poseer conflictos de intereses.

Recibido: 29/10/2017| Aceptado: 06/08/2018

\section{INTRODUCCIÓN}

El número total de muertes por infarto agudo de miocardio con elevación del segmento ST (IAMCEST) ha ido descendiendo ininterrumpidamente durante los últimos 30 años, pero se ha estabilizado en la última década. Según estimaciones de la American Heart Association, el índice de mortalidad a corto plazo oscila entre el 5 y $6 \%$ durante la hospitalización inicial y entre el 7 y $18 \%$ al cabo de 1 año ${ }^{1}$

En nuestro país no disponemos de datos fehacientes que nos permitan saber la incidencia anual de los IAMCEST. La última aproximación y solo de carácter deductivo estimó 42.025 paciente en el año 2005, con una incidencia de 10,8 por 10.000 habitantes de todas las edades y de 24,5 por 10.000 habitantes en mayores de 35 años. En la actualidad se está llevando a cabo, en una tarea conjunta entre la Sociedad Argentina de 


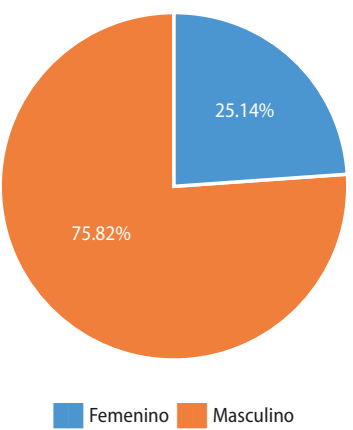

Figura 1. Prevalencia según sexo.

Cardiología, la Federación Argentina de Cardiología y el Ministerio de Salud de la Nación, el Registro Nacional Permanente de Enfermedades Cardiovasculares para el Monitoreo de Políticas Públicas, comenzando por el Estudio del Infarto Agudo de Miocardio con Elevación del ST (ARGEN-IAM-ST); que tiene como objetivo estimar el número de infartos y las características globales de su atención. Los datos se obtienen a través de reportes de encuestas y relevamientos de redes institucionales habitualmente con actividad académica y por lo tanto con sesgos que impiden proyectar estos datos a toda la comunidad. En esta fase del registro se han incluido 1.759 pacientes en 247 centros de todo el país (2015). La conclusión observada y publicada (ARGEN-IAM-ST) fue una mayor prevalencia de tabaquismo (TBQ), un mayor uso de angioplastia primaria con menor indicación de reperfusión con fibrinolíticos, y una reducción significativa del shock cardiogénico en los últimos 5 años. Las variables predictivas de mayor riesgo para el desarrollo de enfermedad cardiovascular incluyen edad avanzada, sexo masculino, dislipemia (DLP), diabetes mellitus (DBT), TBQ, hipertensión arterial (HTA) e historia familiar de enfermedad coronaria².

A pesar de los grandes avances en el conocimiento de la fisiopatología del infarto agudo de miocardio con elevación del segmento ST (IAMCEST), aún se desconocen muchas de las variables que influyen en el desenlace de esta patología y en particular la relación entre la anatomía coronaria afectada y el sexo. En Argentina no existen bases de datos que evalúen este punto en particular; es por ello que este estudio tiene por objetivo analizar la población de un hospital privado de la provincia de Mendoza con diagnóstico de IAMCEST con la intención de establecer una relación directa entre la arteria afectada y el sexo.

\section{MATERIALES Y MÉTODOS}

Se realizó un estudio observacional, analítico, retrospectivo y unicéntrico desde enero de 2014 hasta julio de 2017. Se incluyeron pacientes con diagnóstico de IAMCEST definido según la tercera definición de infarto como detección de un aumento y/o descenso de los valores de biomarcadores cardiacos (preferiblemente troponina cardíaca), con al menos uno de los valores por encima del percentil 99 del límite de referencia superior, y al menos uno de los siguientes parámetros: síntomas de isquemia, cambios significativos en el segmento ST nuevos, bloqueo de rama izquierda nuevo, desarrollo de ondas Q patológicas en el ECG, evidencia por imagen de pérdida de miocardio viable de nueva aparición o anomalías regionales en la motilidad de
Tabla 1. Factores de riesgo cardiovasculares y comorbilidades según sexo.

\begin{tabular}{lcccccc} 
& & \multicolumn{2}{c}{ Femenino } & \multicolumn{2}{c}{ Masculino } & p \\
Edad (años) & Media \pm DE & \multicolumn{2}{c}{$69,79(13)$} & 61,41 & $(12.4)$ & 0,001 \\
DLP & $\mathrm{n} ; \%$ & 8 & $25.0 \%$ & 34 & $31.8 \%$ & 0,46 \\
TBQ & $\mathrm{n} ; \%$ & 14 & $35.9 \%$ & 62 & $53.0 \%$ & 0,64 \\
HTA & $\mathrm{n} ; \%$ & 31 & $79.5 \%$ & 72 & $61.5 \%$ & 0,04 \\
DBT & $\mathrm{n} ; \%$ & 14 & $35.9 \%$ & 25 & $21.4 \%$ & 0,07 \\
Cardiopatía & $\mathrm{n} ; \%$ & 6 & $15.4 \%$ & 24 & $20.5 \%$ & 0,48 \\
isquémica & $\mathrm{n} ; \%$ & 11 & $28.2 \%$ & 34 & $29.1 \%$ & 0,91 \\
Obesidad & $\mathrm{n} ; \%$ & 14 & $35.9 \%$ & 8 & $6.8 \%$ & $<0,0001$ \\
Hipotiroidismo & $\mathrm{n} \% \%$ & 1 & $2.6 \%$ & 4 & $3.5 \%$ & 0,78 \\
\hline ERC & & & & & &
\end{tabular}

DLP: dislipemia. TBQ: Tabaquismo. HTA: hipertensión arterial. DBT: diabetes. ERC: enfermedad renal crónica.

la pared de nueva aparición e identificación de un trombo intracoronario mediante angiografía o autopsia; de menos de 12 horas de evolución a los que se le realizó cinecoronariografía de urgencia ${ }^{3,4}$.

Se excluyeron pacientes con infartos de miocardio sin elevación del segmento ST, IAMCEST de más de 12 horas de evolución, a los que recibieron trombolíticos como estrategia de reperfusión y aquellos derivados de otros centros.

\section{ANÁLISIS ESTADÍSTICO}

Los datos remitidos se incorporaron a una base de datos de Excel. Se analizaron variables como sexo, edad, factores de riesgo cardiovascular, arteria culpable, tipo de tratamiento. Las variables categóricas se expresaron mediante números y porcentajes, y las continuas, según su distribución, como media o mediana, con su correspondiente desvío estándar o intervalo intercuartílico. Para evaluar la asociación entre las variables continuas se utilizó el test de $U$ de Mann-Whitney o test de T, y para las categóricas se utilizó el test exacto de Fisher o Chi Cuadrado, según correspondiera.

\section{RESULTADOS}

Se incluyeron 153 pacientes. La media de edad fue 63,31 años (DE: $\pm 12,99$ ). El $75,82 \%$ de los pacientes enrolados fueron hombres y el 24,14\% mujeres (Figura 1)

El factor de riesgo cardiovascular más prevalente en ambos grupos fue la hipertensión arterial, siendo 79,5\% en mujeres y $61,5 \%$ en hombres ( $p=0,04)$; seguido por tabaquismo (35,9\% mujeres y $53 \%$ hombres; $p=0,64)$, diabetes mellitus $(35,9 \%$ mujeres y $21,4 \%$ hombres; $p=0,07)$ y obesidad $(28,2 \%$ mujeres y $29,1 \%$ hombres; $p=0,91)$. Hubo una mayor prevalencia de hipotiroidismo en el sexo femenino (mujeres $35,9 \%$ y hombres 6,8\% p<0,0001). El número de pacientes con antecedentes de cardiopatía isquémica fue mayor en el sexo masculino, del 20,5\%, y del 15,4\% en mujeres, diferencia no estadísticamente significativa $(p=0,48)$. Se observó un porcentaje pequeño de pacientes con enfermedad renal crónica en ambos sexos (2,6\% mujeres y 3,5\% hombres; $p=0,78$ ) (Tabla 1 ).

No hubo diferencias en la arteria afectada según sexo $(p=0,38)$. Se observó que la arteria culpable más prevalente fue la descendente anterior (DA) sin encontrar diferencias cuando se comparó según sexo: $39,5 \%$ en mujeres y $37,9 \%$ en hombres. Resultados similares se observaron con la arteria coronaria derecha (CD), con una prevalencia li- 


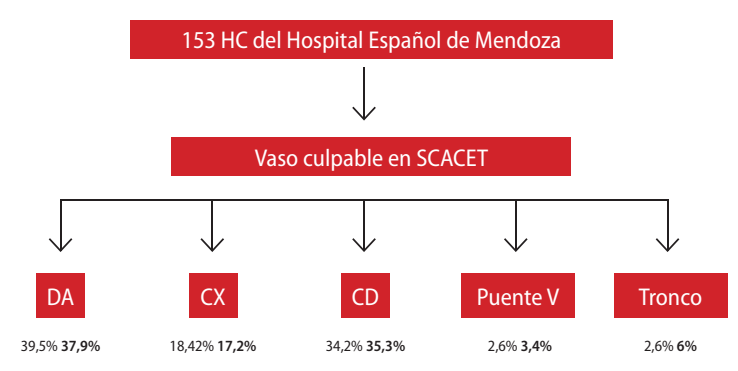

Figura 2. Prevalencia de arteria culpable según sexo. Los porcentaje en fuente regular corresponden al sexo femenino y en negrita al sexo masculino. HC: historia clínica. SCACEST: síndrome coronario agudo con elevación del ST. DA: arteria descendente anterior. CX: arteria circunfleja. CD: arteria coronaria derecha, PUENTE V: puente venoso. Cabe mencionar que la sumatoria de los porcentajes de arteria afectada en mujeres es mayor al 100\% porque en una paciente se identificó a la CD y la Cx como arterias culpables.

geramente menor que la DA (34,2\% mujeres y $35,3 \%$ hombres); la arteria circunfleja (CX) fue el vaso culpable en $18,42 \%$ sexo femenino y $17,2 \%$ sexo masculino. El 2,6\% de las mujeres tuvo compromiso de tronco coronario izquierdo (TCl) siendo mayor el porcentaje de hombres con compromiso se este vaso: $6 \%$. El puente venoso tuvo una baja prevalencia sin diferencias entre sexo (2,6\% mujeres y 3,4\% hombres)

(Figura 2 y 3). Cabe mencionar que la sumatoria de los porcentajes de arteria afectada en mujeres es mayor al 100\% dado que en una paciente se identificó la CD y la Cx como arterias culpables.

\section{DISCUSIÓN}

En nuestro estudio observamos que la prevalencia de IAMCEST es mayor en el sexo masculino, y el principal factor de riesgo es la HTA, seguida por TBQ, DBT y obesidad. Las mujeres presentaron mayor edad y prevalencia de HTA e hipotiroidismo. En cuanto a la arteria responsable, el vaso que más se encontró afectado fue la DA (38,56\%), y con un porcentaje similar la CD (35,94\%). Comparando según sexo no se encontraron diferencias estadísticamente significativas entre ambos grupos $(p=0,33)$. Pero observó una tendencia a mayor compromiso de $\mathrm{TCl}$ entre los hombres.

Estos datos obtenidos coinciden con los publicados en la Revista

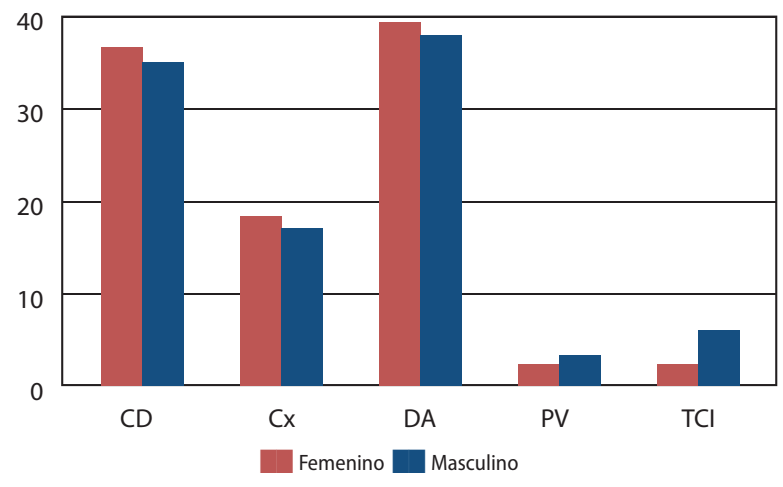

Figura 3. Prevalencia de arteria según el sexo. CD: coronaria derecha. CX: circunfleja. DA: des cendente anterior. PV: puente venoso. TCl: tronco coronaria izquierda.

Cubana de Cardiología en el año 2011, de un registro de 659 pacientes con IAMCEST. El 75,6\% eran hombres y $24,4 \%$ eran mujeres, la distribución de arteria culpable según el sexo fue $77,7 \%$ en los hombres y $22,3 \%$ en las mujeres en la DA; $75,7 \%$ y $22,4 \%$ en la $\mathrm{Cx} ; 75,2 \%$ y $24,8 \%$ en la CD. Al igual que en nuestro estudio, se observó una mayor prevalencia en el TCl en hombres: 88,9 vs. 11,1\% $(p=0,034)^{5}$.

Como limitación de nuestro estudio consideramos que los datos fueron obtenidos de nuestro centro que, aclaramos, es privado. Por ello, los resultados pertenecen a un grupo poblacional sesgado. Si bien reconocemos el aporte a las políticas de salud, exhortamos al resto de la comunidad cientíica y al estado a establecer datos estadísticos fluidos y globales sobre infarto agudo de miocardio en la República Argentina. Es por esto nuestro compromiso para ampliar la base de datos y continuar con futuros aportes.

\section{CONCLUSIÓN}

Más del 75\% de los infartos de miocardio con elevación del segmento ST eran hombres. Las mujeres presentaron un perfil de riesgo cardiovascular diferente, eran más añosas y en ellas era más prevalente la HTA. No hubo diferencias en la arteria coronaria afectada según el sexo. Se observó una tendencia no estadísticamente significativa a mayor enfermedad de TCl en el hombre.

\section{BIBLIOGRAFÍA}

1. O'Gara PT, Kushner FG, Ascheim DD, Casey DE Jr, Chung MK, de Lemos JA, et al 2013 ACCF/AHA Guideline for the Management of ST-Elevation Myocardial Infarction: Executive Summary. Circulation 2013;127(4):e362-425.

2. Federación Argentina de Cardiología (FAC), Sociedad Argentina de Cardiología (SAC), Centro de Teleinformática de FAC (CETIFAC). Relevamiento Nacional permanente de Enfermedades Cardiovasculares para el monitoreo de políticas públicas. Estudio piloto de Infarto Agudo de Miocardio con elevación del ST (ARGEN-IAM-ST) Sociedad Argentina de Cardiología, la Federación Argentina de Cardiología y el Ministerio de Salud de la Nación. Rev Fed Arg Cardiol 2014:43(4):197-201.

3. Steg G, James SK, Atar D, Badano L, Blomstrom Lundqvist C, Borger M, et al. Guía de práctica clínica de la ESC para el manejo del infarto agudo de miocardio en pacientes con elevación del segmento ST. Rev Esp Cardiol 2013;66(1):53.e1-e46.

4. Thygesen K, Alpert JS, Jaffe AS, Simoons ML, Chaitman BR, White HD. Documento de consenso de expertos. Tercera definicion universal del infarto de miocardio. Rev Esp Cardiol 2013;66(2):132.e1-e15.

5. Sánchez Torres N, Pérez del Todo JM, Del Pino Sánchez E, Martínez García G, Suárez Rivero A, Guevara Mirabal G. Caracterización por sexo de los pacientes con sindrome coronario agudo con elevación del segmento ST sometidos a intervencionismo coronario. Rev Cubana Cardiol Cir Cardiovasc 2011;17(3):225-33.

6. Chacón-Diaz 1, Vega A, Aráoz O, Ríos P, Baltodano R, Villanueva F et al. Epidemiological characteristics of ST-segment elevation myocardial infarction in Peru: Results of the PEruvian Registry of ST-segment Elevation Myocardial Infarction (PERSTEMI). Arch Cardiol Mex 2018,88(5):403-12. 